\title{
Avaliação econômica em decorrência da poluição do ar na saúde humana causada pelo impacto da instalação de uma Termoelétrica a gás natural: um estudo de caso para as cidades de Lorena, Canas e Cachoeira Paulista [SP]
}

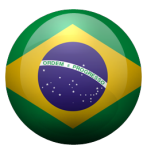

\section{Dante Bachi Junior}

Mestre em Ciências em Engenharia de Energia [Universidade Federal de Itajubá]. Pesquisador do Grupo de Estudos em Energias Renováveis [UNIFEI]. Possui graduação em Petróleo e Gás [Módulo Centro Universitário], e Técnico em Edificações [Mackenzie]. Caraguatatuba [SP] Brasil. <dantejunior@ninhodofalcao.com.br>

\section{Resumo}

0 desenvolvimento a qualquer custo, nos dias atuais, não é mais aceito pela sociedade, com isso as decisões administrativas e econômicas dos gestores públicos, necessitam de ferramentas atualizadas de gestão, diante disso, o presente estudo apresenta uma análise teórica de alguns métodos de valoração econômica ambiental, a monetarização do custo da poluição do ar possibilitando enxergar o custo real ambiental, servindo como instrumento para a sociedade exigir dos empreendedores investimentos em equipamentos de controle de poluentes melhorando a qualidade ambiental e ao mesmo tempo a própria sociedade entenda o ganho econômico em escala. Inicia-se o estudo por meio da definição dos efluentes lançados no ar pelas usinas termelétricas a gás natural, com ênfase no fator recursos naturais; posteriormente tratando sobre a teoria do valor econômico total dos recursos naturais. Em seguida o estudo converge para a análise dos métodos de custos de função demanda, função de produção e doseresposta, e a confrontação da disposição a pagar (DAP) e a disposição a aceitar (DAA) com a avaliação do impacto econômico exercidos pelas doenças respiratórias. 0 estudo encerra-se com a determinação dos custos das doenças associadas à poluição do ar, ou seja, doenças respiratórias no município de Lorena, Cachoeira Paulista e Canas no Vale do Paraíba-SP.

\section{Palavras-chave}

Usina termelétrica. Poluição do ar. Funções dose-resposta. Valoração ambiental.

\section{Economic evaluation as a result of air pollution in human health caused by the installation impact of a thermoelectric plant on natural gas: a case study for the cities of Lorena, Canas and Cachoeira Paulista, SP, Brazil}

\begin{abstract}
Development at any cost, nowadays, is no longer accepted by society, thus the administrative and economic decisions of public managers need updated management tools, on this, the present study presents a theoretical analysis of some valuation methods economic environment, the monetization of the cost of air pollution permitting see the actual cost environment, serving as a tool for society of entrepreneurs require investments in pollution control equipment improving environmental quality while society itself understands the economic gain in scale. Begins the study by defining the effluents discharged into the air by power plants to natural gas, with emphasis on natural resource factor and subsequently treating the theory of total economic value of natural resources. Then the study converges to the analysis of the methods of cost function demand, production function and dose-response, and the confrontation of willingness to pay (WTP) and willingness to accept (DAA) with the assessment of the economic impact exerted by respiratory diseases. The study concludes with the determination of the costs of diseases associated with air pollution, ie respiratory diseases in the city of Lorraine, Cachoeira Paulista and Canas in the Paraíba Valley SP.
\end{abstract}

\section{Keywords}

Thermoelectric plant. Air pollution. Dose-response functions. Environmental valuation. 


\section{Introdução}

O ar possui várias formas de uso, como o uso metabólico natural pelos seres humanos, animais e vegetais, atuando também como receptor e meio de transporte dos resíduos das atividades realizadas pelo homem. Assim, a poluição do ar surge a partir do momento em que o ar, dada a sua disponibilidade, é utilizado de forma não controlada principalmente em áreas geográficas limitadas ou confinadas (Kuwaraha \& Gomes, 2008).

Kuwaraha e Gomes (2008) definem externalidades como os resultados das atividades de produção e consumo que implicam em benefícios ou custos sobre outros agentes, mas que não são refletidos diretamente no mercado, configurando-se como falha no sistema de preços. As externalidades podem aparecer entre consumidores, produtores ou entre consumidores e produtores e ser positivas ou negativas. Externalidades negativas são aquelas resultantes da ação de uma das partes que impõe custos à outra. 0 uso indiscrimina-do do recurso natural "ar", as ações que geram a sua poluição, bem como de outros recursos naturais, podem ser considerados exemplos de externalidades negativas.

Externalidades necessitam de correção, haja vista consistirem falhas no sistema de preços, comprometendo a capacidade de decisão dos agentes econômicos. A existência da poluição do ar configura uma externalidade negativa, fazendo necessária a adoção de um instrumento de política ambiental, que deve ser aplicado corretamente, tarefa não trivial aos gestores de política ambiental. A dificuldade na adoção do instrumento correto deve-se ao fato de que recursos naturais como o ar são bens de propriedade comum, que não têm direitos de propriedade bem definidos e podem ser utilizados sem a necessidade de pagamento pelo uso do recurso (Kuwaraha \& Gomes, 2008).

A existência de um grande número de empresas e indivíduos envolvidos em mercados onde há a presença de externalidades, somada à existência de bens de propriedade comum, imprime ao governo papel fundamental como agente promotor de acordo entre as partes, de forma que haja a internalização das externalidades, ou seja, para que os custos dos problemas ambientais sejam internalizados (Kuwaraha \& Gomes, 2008), favorecendo processos onde a sustentabilidade possa ser alcançada.

Ainda de acordo com estes autores, vários são os instrumentos que ajudam a gerir os problemas ambientais, podendo ser instrumentos de políticas que atuam sobre quantidades, tais como os de comando e controle, ou que atuam sobre custos, como os incentivos econômicos. As políticas de comando e controle são aquelas que não permitem outras soluções para o agente econômico na solução do problema ambiental, ou seja, não é um mecanismo que permite uma flexibilidade na tentativa de encontrar soluções ambientais e, via de regra, são determinadas legalmente.

Essas políticas são realizadas via regulação direta e indireta, adoção de legislação e normas, sendo as regulações no Brasil dividi-das basicamente em quatro categorias, a saber: padrões ambientais de qualidade e de emissão, controle do uso do solo (saneamento e áreas de proteção), licenciamento que exige estudo e relatório de impacto ambiental (EIA e RIMA, respectivamente) e através de multas ou compensações, como forma de penalidades. Os instrumentos previstos pela política de comando e controle são os padrões de emissão, o zoneamento, as cotas não transferíveis e o controle equipamentos, processos e insumos (Kuwaraha \& Gomes, 2008).

(Kuwaraha \& Gomes, 2008) afirmam ainda que a solução para a problemática ambiental deverá ter como objetivo a junção de políticas que atendam aos critérios de eficiência, na busca de um menor custo, e ao critério de efetividade, de forma a atingir os objetivos ambientais desejados. A definição de objetivos ambien-tais, porém, depende da forma como a sociedade define seus próprios objetivos, ou, da forma como o problema ambiental é enfrentado por esta sociedade em termos de ordenação de prioridades.

\section{Aspectos técnicos da poluição do ar}

Entre as principais fontes de poluição do ar encontram-se os combustíveis fósseis que atuam como agentes poluentes. Segundo Baird (2002), "A introdução de substâncias oxigenadas, que são 
hidrocarbonetos nos quais alguns átomos foram substituídos por oxigênio, na gasolina americana, tem como objetivo reduzir as emissões de CO dos veículos". Assim, Baird (2002) afirma que a introdução das substancias oxigenadas teve resultados significativos, como os níveis de concentração externa de monóxido de carbono nos Estados Unidos que foram reduzidos em $37 \%$ entre os anos de 1986 e 1995. No Brasil, a composição da gasolina inclui uma parcela de álcool que, na qualidade de substância oxigenada, alcança os mesmos objetivos que a similar norte-americana (Kuwaraha \& Gomes, 2008).

O dióxido de carbono, $\mathrm{CO} 2$, é um composto proveniente da combustão completa de combustíveis fósseis e resultado do processo de respiração aeróbia dos seres vivos. Entre os demais poluentes presentes em ambientes, podemos encontrar os óxidos de nitrogênio, NOx, como o óxido de nitrogênio, NO, e o dióxido de nitrogênio, NO2, sendo em grande parte originados, também, a partir de processos de combustão (Kuwaraha \& Gomes, 2008).

As partículas respiráveis também pertencem ao grupo de poluentes presentes em ambientes. 0 Material particulado é um dos poluentes químicos previstos pela ANVISA (2003). (Kuwaraha \& Gomes, 2008) definem matéria particulada os poluentes do ar como partículas como pólen, poeira, fuligem e as partículas de óleo, partículas provenientes de materiais líquidos ou sólidos e que podem permanecer em suspensão, originadas a partir de fenômenos naturais (no caso da dispersão do pólen ou suspensão de material particulado em razão da ação do vento) e do processo de combustão (fuligem e partículas de óleo).

A poluição do ar na Região Metropolitana de São Paulo é decorrente das emissões atmosféricas de cerca de 2.000 indústrias e da frota de aproximadamente 8,4 milhões de veículos automotores, composta por 7,0 milhões de veículos do ciclo Otto, 460 mil veículos a diesel e 1.020 mil motos, representando cerca de $1 / 5$ do total nacional. De acordo com as estimativas de 2007, essas fontes são responsáveis pela emissão de 1,5 milhão de t/ano de CO, 365 mil t/ano de HC, 339 mil t/ano de NOx, 29,5 mil t/ano de MP total e 8,2 mil t/ano de SOx (CETESB, 2007). Já o controle e fiscalização das emissões atmosféricas industriais têm como objetivo atingir os padrões de qualidade do ar. Controlar a emissão de poluentes industriais na atmosfera requer, dentre outras medidas, a realização de análises periódicas e implementação de medidas preventivas. E para o caso especifico dos NOx, os padrões de emissão para as fontes, são exigidos, conforme o Art. 41 e Parágrafo único do Decreto 8.468/76, que dispõe sobre a prevenção e o controle da poluição e estabelece as atribuições da CETESB (SMA, 1976).

\section{Emissão de poluentes atmosféricos de usinas termelétricas e seus impactos no meio ambiente e na saúde}

Os efeitos das emissões atmosféricas são diversos, atuando de forma local, regional e global. Os impactos locais são limitados ao entorno das fontes emissoras. Já os impactos regionais compreendem um raio bem maior, de centenas de quilômetros. Os impactos globais afetam todo o planeta, um exemplo é o efeito estufa e aquecimento global (Goldemberg, 2010).

Segundo Veiga (2001), a emissão de poluentes para a atmosfera pode ser considerada uma das principais ações impactantes resultante do funcionamento de usinas termoelétricas, pois consiste na emissão de material particulado e gases provindos do processo de combustão, cuja quantidade e composição variam conforme a tecnologia e o combustível utilizado. Os principais poluentes emitidos desses empreendimentos são MP, SOx, COx, NOx e compostos orgânicos voláteis.

O material particulado (MP) é considerado qualquer substância (exceto água pura) existente na forma líquida ou sólida na atmosfera com dimensões microscópicas porém, maiores que as dimensões moleculares. Assim, essas partículas classificam-se em finas (diâmetro menor que $2,5 \mu \mathrm{m}$ ) e grossas (diâmetro maior que 2,5 $\mathrm{m}$ ). As partículas com diâmetro menor que $10 \mu \mathrm{m}$ (PM10) são as que mais causam efeitos adversos à saúde humana, pois tendem a se depositar nas regiões traquio-bronquial e pulmonar (Veiga, 2001).

A Tabela 1 mostra as emissões de poluentes atmosféricos como SO2, NOx, CO2 e MP do funcionamento de usinas termoelétricas que utilizam como combustível: óleo combustível e gás natural.

(C) Labor \& Engenho, Campinas [SP] Brasil, v.10, n.4, p.402-412, out./dez. 2016. 
Tabela 1. Emissões atmosféricas de termoelétricas (1.000 MW), por combustível.

\begin{tabular}{|c|c|c|c|c|c|}
\hline \multirow{2}{*}{ COMBUSTivEL } & \multirow{2}{*}{ QUANTIDADE (ton) } & \multicolumn{4}{|c|}{ EMISSÓES ATMOSFÉRICAS ANUAIS } \\
\cline { 3 - 6 } & & SO2 (ton) & NOx (ton) & CO2 (ton) & MP (ton) \\
\hline Óleo combustível (3\%S) & 2.000 .000 & 40.000 & 30.000 & 5.700 .000 & 5.400 \\
\hline Gás natural & 2.000 .000 & 20 & 15.000 & 4.400 .000 & 150 \\
\hline
\end{tabular}

Fonte: Veiga, 2001.

No processo de geração de energia elétrica com gás natural, a combustão é o principal responsável pela emissão de poluentes. Quando as turbinas operam com baixa potência, ocorre maior emissão de hidrocarbonetos e CO. Contudo a emissão de fumaça e NOx é insignificante. Do contrário, quando a potência chega ao máximo, a emissão de HC e CO diminui. No entanto, a emissão de NOx e fumaça é muito alta. Desse modo, a concentração da maioria dos poluentes de interesse nos gases de escape das turbinas a gás pode estar relacionada à temperatura e ao tempo de permanência dos gases na câmara de combustão (Lora, 2004 apud Guena, 2007).

\section{Aspectos metodológicos na avaliação econômica do impacto ambiental}

Segundo Seroa da Motta et al. (1998), os impactos ambientais podem ser estimados por diversas técnicas de mensuração. Busca-se encontrar, para o indivíduo afetado, a sua disposição a pagar (DAP) para evitar um impacto ambiental negativo, ou a disposição a aceitar (DAA) uma compensação quando o impacto negativo já tenha ocorrido. Portanto, deseja-se estimar quanto os indivíduos valoram, em termos monetários, a redução do risco de mortalidade e da incidência das doenças (morbidade) associadas com poluição atmosférica. Essa tarefa pode ser implementada mediante o uso de métodos de valoração econômica encontrados na literatura. Estes métodos são:

- Métodos da Função de Produção: Método da Produtividade Marginal (produção sacrificada); Métodos de Mercados de Bens Substitutos (gastos defensivos, custos evitados, custos de controle).

- Métodos da Função de Demanda: Métodos de Mercado de Bens Complementares; Método dos Preços Hedônicos; Método de Custos de Viagem; Método de Valoração Contingente.

Dentre as metodologias teóricas utilizadas para avaliar monetariamente os efeitos de morbidade e de mortalidade decorrentes da poluição atmosférica, destaca-se o método de valoração contingente.

Conforme Seroa da Motta et al. (1998), o método de valoração contingente busca estimar a disposição a pagar pela redução da incidência de doenças e do risco de morte através de pesquisa direta aos indivíduos, requerendo que estes eliciem suas preferências. Com isto, esse método permite a estimação de valores mais realísticos da DAP. Entretanto, sua desvantagem está nos elevados custos para a condução das entrevistas e outros componentes das pesquisas.

Dadas as dificuldades desta técnica, um recurso recomendado em literatura recente sobre avaliação deste tipo de impacto ambiental é o método da Transferência de Benefícios, que consiste em adaptar a DAP - ou outra estimativa - de outras localidades para o local que está sendo estudado. A transferência de benefícios é a aplicação de [...]

[...] valores monetários de um estudo particular de valoração para um ambiente de decisão política alternativo, ou secundário, freqüentemente em outra área geográfica [diferente] de onde o estudo original foi executado (Markandya, 1998).

Pode-se chegar a valores para as DAP onde não existem estudos, a partir do uso de estimativas encontradas em outras regiões do país, ajustando-as para o caso em estudo, mediante a diferença na renda real per capita entre estes locais e o nosso local de análise.

A transferibilidade depende da capacidade de usar os dados de diferentes estudos e estimar os fatores sistemáticos que resultariam nas diferenças entre as estimativas. A melhor prática é usar estimativas de fontes semelhantes, ou com características aproximadas, àquelas em que o procedimento 
de transferência está sendo aplicado e ajustá-las por diferenças nas variáveis envolvidas, onde isto for possível.

O outro método de valoração adequado para a estimação de custos de saúde é o da Produtividade Marginal na qual se estima a produção sacrificada do trabalhador associada ao dano ambiental. Outra forma é analisar o mercado de bens substitutos, em que se faz a avaliação com base em recursos econômicos que foram direcionados para mitigar os problemas causados pela degradação ambiental. Com esta técnica, estima-se os gastos que foram incorridos para evitar o dano ambiental, a produção e/ou renda deixada de ser criada, bem como os gastos feitos diretamente para sanar o impacto ambiental.

Na avaliação de custos associados à saúde, separa-se o estudo em dois focos: mortalidade e morbidade.

\subsection{Valor presente da produção futura (VPPF)}

Segundo Seroa da Motta et al. (1998), ao avaliar custos associados à saúde, determinado estudo deve ser dividido principalmente em duas etapas, quanto à mortalidade e morbidade causada pela poluição.

A mortalidade é questionada quanto à questão da mudança de risco a vida. Esta é dada pela soma de valores que um indivíduo associa a sua saúde e suas chances de sobreviver e também o que outros indivíduos estariam dispostos a pagar (DAP) para evitar o risco á saúde daquele primeiro indivíduo. Logo, a sociedade incorre em um custo o qual seria evitado se não houvesse determinado risco em relação à saúde do indivíduo em questão.

Estimada a disposição a pagar pela variação do risco de morte em alguma atividade, pode-se encontrar o Valor de uma Vida Estatística (VVE).

$$
V V E=\frac{D A P}{\Delta R i s c o}
$$

Estimado o VVE associado a uma atividade com risco de morte para um indivíduo, pode-se multiplicá-lo por uma variação no risco de vida por outra atividade e estimar a disposição a pagar para evitar mortes por esta outra atividade. Utilizando dados sobre variação de risco de morte, ambos para determinado local obtêm-se o VVE para o local em questão.

Além disso, pode-se utilizar outra forma de avaliar este mesmo impacto negativo, através da abordagem da Produção Sacrificada dos anos de vida perdidos. Esta metodologia admite por pré-suposto fundamental que o valor de uma vida para a sociedade se equivale ao Valor Presente da Produção Futura (VPPF) que seria gerada por tal trabalhador.

$$
\left.V V E \Leftrightarrow V P P F_{i}=\sum_{j=i+1}^{85}\left[\left(P_{i}^{j}\right)_{1}\right] \cdot\left[\left(P_{i}^{j}\right)_{2}\right] \cdot\left[\left(P_{i}^{j}\right)_{3}\right] \cdot\left[Y_{i} \cdot(1+g)\right)^{-i}\right]
$$

VPPFi = valor presente da produção futura ou da renda futura da pessoa de idade i;

$\left.\left[\left(P^{j}\right)_{1}\right)_{1}\right]=$ probabilidade de que a pessoa, com idade i, esteja viva na idade j;

$\left[\left(P_{i}^{j}\right)_{2}\right]=$ probabilidade de que a pessoa, com idade i, esteja na força de trabalho na idade j;

$\left.\left[\left(P^{j}\right)_{3}\right]_{3}\right]=$ probabilidade de que a pessoa, com a idade $\mathrm{i}$, esteja empregada com a idade $\mathrm{j}$;

$g=$ taxa média de crescimento da renda per capita; $Y j=$ renda ou a produção esperada da pessoa na idade i; $r$ = taxa de desconto; 


\subsection{Morbidade (custo da doença)}

A morbidade consiste na real incidência das doenças. No caso deste trabalho trata-se de doenças respiratórias. Na abordagem de Seroa da Mota et al. (1998), os custos da saúde relacionados à poluição atmosférica deveriam ser classificados em quatro categorias, como segue:

a) Gastos médicos associados com tratamento de doenças induzidas pela poluição;

b) Dias de trabalho perdidos resultantes da enfermidade;

c) Gastos para evitar ou prevenir (gastos preventivos) e atividades associadas com tentativas de mitigar a doença;

d) Desutilidade associada com os sintomas e oportunidades de lazer perdidas devido à doença.

Devido à dificuldade de estimar as parcelas relevantes aos dois últimos itens, procurar-se-á mensurar o custo de saúde associado à poluição através da soma dos gastos hospitalares por evento e por faixa etária com o valor dos dias de trabalho perdidos devido às doenças respiratórias. Como fator financeiro será utilizado à renda média por cada faixa etária, com base nos salário mínimo. Por esta razão, os custos totais serão multiplicados por dois, para o cálculo do custo da doença:

$$
\mathrm{CD}=(\text { Gastos Hospitalares }+(\text { Número de Dias Perdidos } *(\text { Renda/30 }))) * 2
$$

\subsection{Análise dos resultados pelo cálculo do VPPE e do CD [custo da doença] nas cidades de Lorena, Canas e Cachoeira Paulista [estado de São Paulo]}

Para calcular o VPPF utilizou a mesma metodologia usada por Seroa da Motta et al. (1998). Desta forma, foram realizados os seguintes procedimentos para a obtenção das estimativas das probabilidades:

$\left[\left(P_{i}^{j}\right)_{1}\right]=1$ - (taxa de mortalidade por faixa de idade) .

$\left[\left(P_{i}^{j}\right)_{2}\right]=$ taxa de participação econômica por faixa de idade.

$\left[\left(P_{i}^{j}\right)_{3}\right]=1-($ taxa de desemprego por faixa de idade).

Para os procedimentos adotam-se os dados considerados no trabalho de Fernandez et al. (2010), pois representam com boa aproximação a realidade das cidades de Lorena, Canas e Cachoeira Paulista.

Para chegar a um valor mais próximo da realidade em relação à renda média por faixa etária Yi, recorreu-se ao seguinte procedimento. Com dados encontrados no Sistema IBGE de Recuperação Automática (SIDRA), conseguiu-se o rendimento por faixa etária para as cidades de Lorena, canas e Cachoeira Paulista. Além disso, os valores encontrados estavam em termos de salários mínimos para o ano de 2010, conforme Tabela 2.

Tabela 2. Valores aproximados do nível de renda - 2010.

\begin{tabular}{l|c|c}
\multicolumn{1}{c|}{ Faixas de Renda } & $\begin{array}{c}\text { Valor } \\
\text { Aproximado }\end{array}$ & Observações \\
\hline Até 1 salário mínimo & $\mathrm{R} \$ 700,00$ & Mediana \\
\hline De 1 a 5 salários mínimos & $\mathrm{R} \$ 1.750,00$ & Mediana $=($ Salário multiplicado x 5) / \\
\hline Acima 6 salários mínimos & $\mathrm{R} \$ 4.200,00$ & Consideraram-se seis salários mínimos \\
\hline Sem rendimento & 0 & \\
\hline
\end{tabular}

Fonte: Elaboração própria com dados do IBGE-SIDRA.

Nas faixas de renda de um até cinco salários, foi então considerado a mediana (valor do meio). 
Logo, multiplicou-se o salário mínimo por cinco e dividiu-se por dois, chegando ao número de $\mathrm{R} \$ 1.750,00$. Para os cidadãos que estavam na faixa de renda superior a cinco salários mínimos, considerou-se uma boa aproximação o valor de seis salários $R \$ 4.200,00$.

A partir dos dados encontrados acima foi necessário agregar o nível de renda por número de moradores como segue na Tabela 3:

\begin{tabular}{|c|c|c|}
\hline Faixa de Renda & Faixa Etária & Hab/Faixa Etária \\
\hline até 1 salário mínimo & 10 a 19 anos & 3325 \\
\hline até 1 salário mínimo & 20 a 24 anos & 2216 \\
\hline até 1 salário mínimo & 25 a 39 anos & 4838 \\
\hline até 1 salário mínimo & Acima de 40 anos & 4731 \\
\hline Mais de 1 a 5 salários mínimos & 10 a 19 anos & 1494 \\
\hline Mais de 1 a 5 salários mínimos & 20 a 24 anos & 3975 \\
\hline Mais de 1 a 5 salários mínimos & 25 a 39 anos & 14208 \\
\hline Mais de 1 a 5 salários mínimos & Acima de 40 anos & 13029 \\
\hline Mais de 5 Salários mínimos & 10 a 19 anos & 28 \\
\hline Mais de 5 Salários mínimos & 20 a 24 anos & 26 \\
\hline Mais de 5 Salários mínimos & 25 a 39 anos & 1782 \\
\hline Mais de 5 Salários mínimos & Acima de 40 anos & 2694 \\
\hline Sem rendimento & 10 a 19 anos & 2830 \\
\hline Sem rendimento & 20 a 24 anos & 1345 \\
\hline Sem rendimento & 25 a 39 anos & 1750 \\
\hline Sem rendimento & Acima de 40 anos & 1159 \\
\hline
\end{tabular}

Tabela 3. Agrupamento de renda por faixa etária - 2010.

Fonte: Elaboração própria com dados do IBGE-SIDRA.

Em suma, com o número total de habitantes de por faixa etária, mostrados na Tabela 4, conforme os dados retirados do IBGE-SIDRA.

\begin{tabular}{|c|c|}
\hline Faixa Etária & Total Habitantes \\
\hline 10 a 19 anos & 19802 \\
20 a 24 anos & 10098 \\
25 a 39 anos & 27775 \\
Acima de 40 anos & 43496 \\
\hline
\end{tabular}

Tabela 4. Número total de habitantes por faixa etária - 2010. Fonte: Elaboração própria com dados do IBGE-SIDRA.

Com estas informações, somou-se o número de habitantes de cada faixa etária dentro de cada grupo de renda e multiplicou-se pelos dados salariais retirados da Tabela 2. Por conseguinte, dividiu-se o valor pelo número total de habitantes da Tabela 4 em cada faixa etária. Cabe destacar, que os habitantes que não possuíam rendimento, a sua renda média foi considerada 


\begin{tabular}{c|c|c}
\hline Faixa Etária & Renda Média 2010 & Renda Média 2013 \\
\hline 10 a 19 anos & $\mathrm{R} \$ 249,32$ & $\mathrm{R} \$ 316,03$ \\
20 a 24 anos & $\mathrm{R} \$ 853,30$ & $\mathrm{R} \$ 1.079,98$ \\
25 a 39 anos & $\mathrm{R} \$ 1.044,07$ & $\mathrm{R} \$ 1.321,43$ \\
Acima de 40 anos & $\mathrm{R} \$ 860,47$ & $\mathrm{R} \$ 1.089,06$ \\
\hline
\end{tabular}

como zero. Mostram-se os resultados na Tabela 5:

Tabela 5. Renda média por faixa etária.

Fonte: Elaboração própria com base em IBGE-SIDRA e SEADE.

A Tabela 5 mostra os valores encontrados para a renda média, destaca-se que estes valores foram atualizados com o IGP-DI fornecido pela SEADE para o mês de Janeiro de 2013.

Para o cálculo da taxa de correção g foram utilizados novamente os resultados do trabalho de Fernandez et al. (2010). Estes chegaram a taxa de aproximadamente 0,02 ou dois por cento para o crescimento da renda nas cidades de Lorena, Canas e Cachoeira Paulista. Assim, utilizaram-se dois valores para descontar o Valor da Produção Futura por faixa de idade. Os valores de $r=3 \%$ e de $r=10 \%$ segundo Seroa da Motta et al. (1998), são os mais comuns adotados na literatura específica da área. É importante salientar que o limite superior da faixa etária, acima de 40 anos leva em conta os indivíduos que possuam 40 anos até a idade de 85 anos. Segue a Tabela 6 com

\begin{tabular}{c|c|c}
\hline Faixa Etária & VPPF $\mathbf{r}=\mathbf{0 , 0 3}$ & VPPF $\mathbf{r}=\mathbf{0 , 1}$ \\
\hline 10 a 19 anos & $\mathrm{R} \$ 59.043,42$ & $\mathrm{R} \$ 8.466,22$ \\
\hline 20 a 24 anos & $\mathrm{R} \$ 44.632,01$ & $\mathrm{R} \$ 9.560,82$ \\
25 a 39 anos & $\mathrm{R} \$ 34.489,36$ & $\mathrm{R} \$ 11.135,28$ \\
40 ou mais & $\mathrm{R} \$ 16.006,39$ & $\mathrm{R} \$ 5.361,65$ \\
\hline
\end{tabular}

os resultados finais:

Tabela 6. Valor Presente da Produção Futura - VPPF: Lorena, Canas e Cachoeira Paulista - 2013. Fonte: Elaboração própria, com base nos dados IBGE-SIDRA e SEADE.

Os valores encontrados para a produção futura relatam o quanto um cidadão local produziria hoje dentro da sua faixa etária até a idade de oitenta e cinco anos. Cabe destacar que os valores encontrados são em termos médios. Também é importante analisar que o parâmetro taxa de mortalidade dentro da análise relacionada é uma boa aproximação do efeito da poluição atmosférica, sendo este responsável pela produção sacrificada. Embora o nível de renda seja maior de acordo com o avanço da faixa etária, em termos médios, a taxa de mortalidade aumenta provocando uma redução na VPPF, como mostra a Tabela 6.

\subsection{Custo das doenças respiratórias}

Para o cálculo do custo gerado na saúde pelas doenças respiratórias foram levantados os dados de gastos hospitalares e o número de internações do sistema DATASUS.

Neste também se obteve o tempo médio de permanência por internação, com a finalidade de inferir o número de dias perdidos de trabalho. Tal índice foi obtido, multiplicando-se o número de internações pelo tempo medido de internação, os dados foram utilizados do ano de 2009, com exceção da renda por faixa etária que já está atualizada para o Janeiro do ano de 2013.

Com a soma dos gastos hospitalares com o valor calculado dos dias de trabalho perdidos do ano de 2009, chegou-se ao custo da doença (CD) associada à poluição do ar nas cidades de Lorena, 
Canas e Cachoeira Paulista, como mostra a Tabela 7:

Tabela 7. Custo das doenças respiratórias associadas à poluição do ar - 2009.

\begin{tabular}{c|c|c|c|c}
\hline Faixa Etária & Gastos Hospitalares & Morbidade (*) & Renda Mensal & Custo das Doenças \\
\hline $10-17$ & $\mathrm{R} \$ 14.498,80$ & 1966,8 & $\mathrm{R} \$ 316,03$ & $\mathrm{R} \$ 70.435,45$ \\
$18-24$ & $\mathrm{R} \$ 20.717,96$ & 3814,25 & $\mathrm{R} \$ 1.079,98$ & $\mathrm{R} \$ 316.056,83$ \\
$25-39$ & $\mathrm{R} \$ 23.407,07$ & 5512,5 & $\mathrm{R} \$ 1.321,43$ & $\mathrm{R} \$ 532.439,67$ \\
40 ou mais & $\mathrm{R} \$ 317.035,72$ & 84259,59 & $\mathrm{R} \$ 1.089,06$ & $\mathrm{R} \$ 6.751 .654,71$ \\
\hline
\end{tabular}

Fonte: Elaboração Própria, com dados retirados do DATASUS.

Notas: Custo das Doenças $=\{\text { Gastos Hospitalares }+[\text { Morbidade } *(\text { renda } / 30)]\}^{*} 2$

Morbidade $\left({ }^{*}\right)=$ Número de Dias Perdidos * Tempo Médio de Permanência por Internação.

\section{Conclusão}

Os dados da Tabela 7 mostram os custos relacionados às doenças respiratórias nos municípios de Lorena, Canas e Cachoeira Paulista. É pode-se dizer, que as doenças do aparelho respiratório tenham como efeito direto a poluição do ar. Sendo assim, o custo da doença é considerado como externalidades negativas sofridas pela população das cidades, e este custo também pode ser considerado como uma forma de quantificar o quanto se deixa de produzir pelos efeitos sofridos pela sociedade.

Quanto à perda de produção devido aos efeitos da poluição do ar, é importante destacar que a taxa de mortalidade capta o efeito da poluição do ar na vida dos habitantes. Embora a renda seja maior com o decorrer das faixas etárias, a taxa de mortalidade, provocando uma redução no valor total da produção significativa em todas as faixas etárias.

E ainda, o cálculo da morbidade pelo valor do custo da doença mostra a relação do valor que a população paga efetivamente pelas doenças respiratórias, causadas pela poluição ar. Logo, as externalidades negativas recebem a monetarização de acordo com este montante pago. Desta forma, pode-se, mensurar custos sociais a serem internalizados pelas empresas fontes das emissões.

Com relação ao estudo de caso proposto neste trabalho para melhor compreensão do método de valoração contingente: A implantação de um termoelétrica a gás natural na região de Lorena, Canas e Cachoeira Paulista, no estado de São Paulo, com capacidade 360MW, gerado em uma turbina a gás, e $190 \mathrm{MW}$ gerado em uma turbina a vapor, com consumo previsto de $49.283,25 \mathrm{~m}^{3} / \mathrm{h}$ a $20^{\circ} \mathrm{C}$ e $1 \mathrm{~atm}$. E sendo mantidos os níveis de emissões de efluentes aéreos, esses informados pela empresa responsável, a Termo São Paulo, no EIA/RIMA do empreendimento. 0 impacto na saúde das populações provocado pela poluição do ar será baixo. E isso fica claro a medida, em que compara-se os valores dos gastos com as doenças do aparelho respiratório da região em estudo com outros municípios e outras regiões do estado de São Paulo, essas detentoras de volumes de poluentes atmosféricos bem mais agudos, conforme: Figuras 1 e 2.

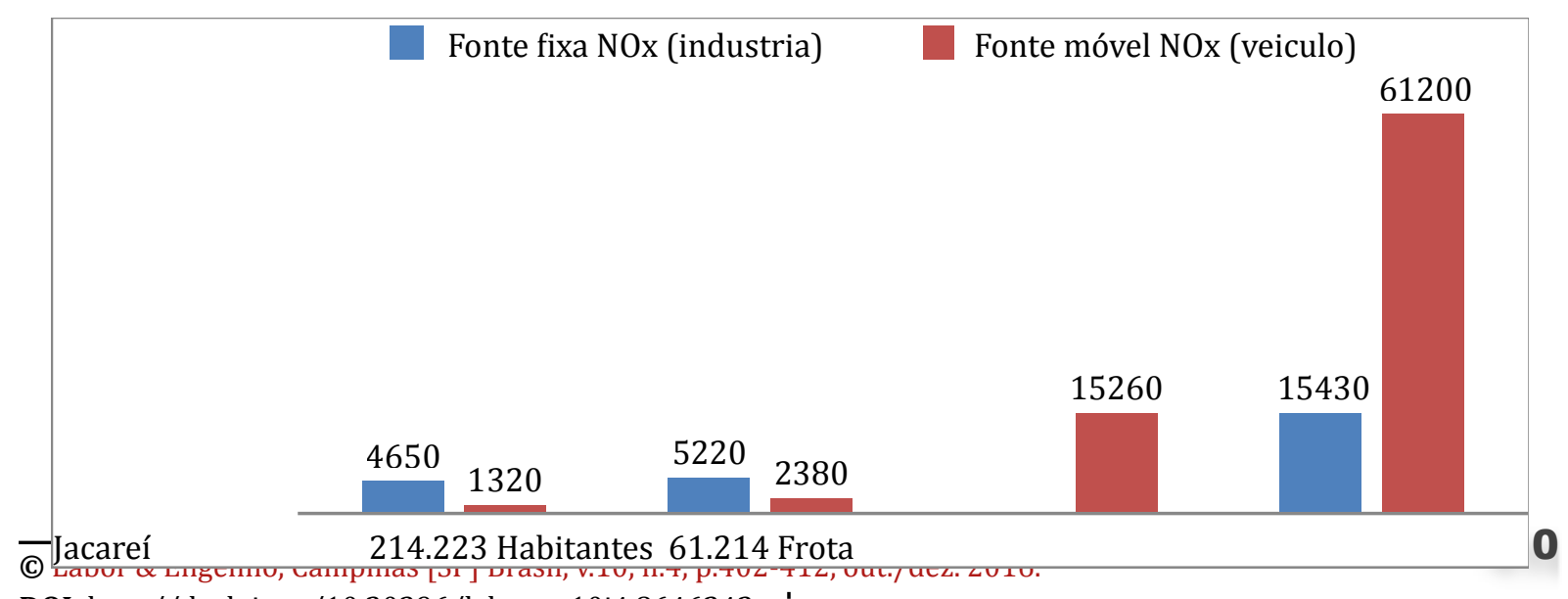

DOI: http://dx.doi.org/10.20396/labore.v10i4.8646242 
Morbidade $2009 \quad$ Mortalidade 2008

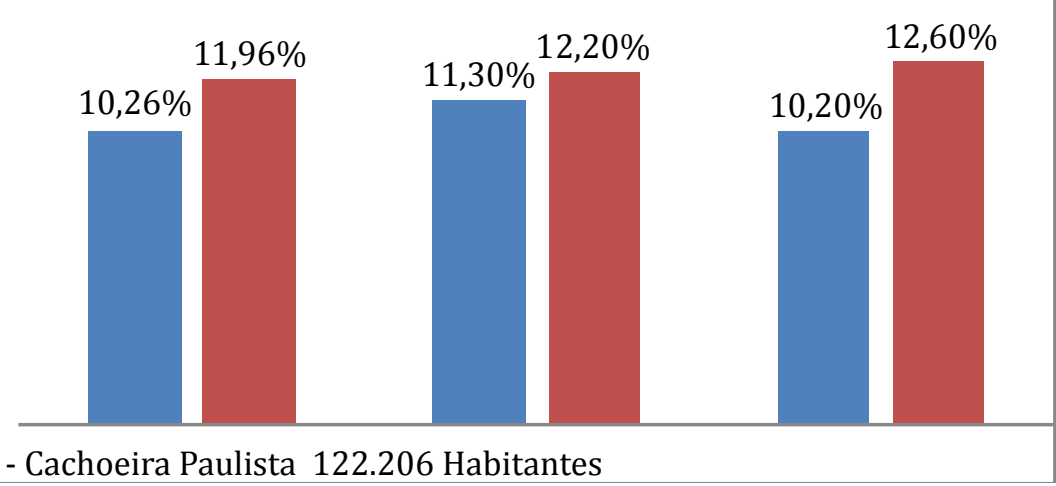

Lorena - Canas - Cachoeira Paulista 122.206 Habitantes

Figura 1. Gráfico de emissões de NOx por regiões do estado de São Paulo.

Figura 2. Gráfico de doenças do aparelho respiratório por municípios.

Fonte: SIH/SUS. Situação da base de dados nacional em 03/05/2010.

\section{Referências}

Abdallah, P. R., Fernandez, R. N., \& Menezes, G. (2010). Custo das doenças respiratórias associadas á poluição atmosférica: Um estudo de caso para a cidade do Rio Grande.

Baird, C. (2002). Química ambiental (2a ed.) (M.A. Lobo Recio, \& L.C.M. Carrerá, Trads.). Porto Alegre: Bookman.

Castro (2003). Questões metodológicas para a investigação dos efeitos da poluição do ar na saúde. Revista Brasileira de Epidemiologia, 6, 135-149.

Cetesb (s.d.). Relatório de Qualidade do Ar no Estado de São Paulo. Recuperado em abril, 2013, de http:// www.cetesb.sp.gov.br/ar/qualidade-do-ar/31-publicacoes-e-relatorios . 2013. Acesso em: Abr. 2013.

Coelho, M. de S. Z. S. (2007). Uma análise estatística com vistas a previsibilidade de internações por doenças respiratórias em função de condições meteorológicas na cidade de São Paulo. Tese de Doutorado, Instituto de Astronomia, Geofísica e Ciências Atmosféricas (IAG), Universidade de São Paulo, São Paulo, SP, Brasil.

Duchiade, M. P. (1992). Poluição do ar e doenças respiratórias: uma revisão. Caderno de Saúde Pública, 8 (3), 311-330.

Gomes, M. J. M. (2002). Ambiente e pulmão. Jornal de Pneumologia, 28, 261-269.

Goldemberg, J. (2010). Energia e Desenvolvimento Sustentável. (Coleção Sustentabilidade Vol.4). São Paulo: Blucher.

Góralcyk, M. (2003). Life-cycle assessment in the renewable energy sector. Applied Energy, 75, 205-211.

Gouveia, N., \& Fletcher, T. (2000). Respiratory diseases in children and outdoor air pollution in São Paulo, Brazil: a time series analysis. Journal of Occupational and Environmental Medicine, 57, 477-483.

Gouveia, N., Mendonça, G. A. E S, Leon, A. P. de, Correia, J. E. de M., Junger, W. L., Freitas, C. U. de, Daumas, R. P., Martins, L. C., Giussepe, L., Conçeição, G. M. S., Manerich, A., \& Cunha-Cruz, J. (2003). Poluição do ar e efeitos na saúde nas populações de duas grandes metrópoles brasileiras. Epidemiologia e Serviços de Saúde, 12, 29-40.

Guena, A.M. de 0. (2007). Avaliação ambiental de diferentes formas de geração de energia elétrica. Dissertação de Mestrado, Universidade de São Paulo (Instituto de Pesquisas Energéticas e Nucleares), São Paulo, SP, Brasil. 
Heintz, R. J., \& Tol, R. S. J. (1996). Secondary benefits of climate control policies: implications for the global environment facility [CSERGE Working Paper GEC 96-17]. Centre for Social and Economic Research on the Global Environment, University of East Anglia, London.

Kuwahara, M. Y., \& Gomes, A. B. O. (2008). Custos de saúde associados à poluição do ar em ambientes internos: em busca de aproximações transdisciplinares. Anais do VI ENABER - São Paulo. Recuperado em abril, 2013, de http://docplayer.com.br/11451932-Custos-economicos-da-poluicao-do-ar-em-ambientes-internos-em-buscade-aproximacoes-transdisciplinares-1-resumo.html.

Markandya, A. (1998). The valuation of health impacts in developing countries [Internal Paper]. University of Bath, Department of Economics and International Development. Bath, U.K.

Medeiros, A. M. (2003). Bases metodológicas para a incorporação da variável ambiental no planejamento da expansão termelétrica no Brasil. Dissertação de Mestrado (Engenharia), Universidade Federal do Rio de Janeiro, Rio de Janeiro, RJ, Brasil.

Serôa da Motta, R. (1995). Contabilidade Ambiental: Teoria, Metodologia e Estudos de Casos no Brasil. Rio de Janeiro: Instituto de Pesquisa Econômica Aplicada.

Serôa da Motta, R., \& Mendes, A. P. (1995). Custos da saúde associados à poluição de ar no Brasil. Pesquisa e Planejamento Econômico, 25 (5).

Veiga, J. R. C. da. (2001). Oportunidades de negócio com a repotenciação de usinas: aspectos técnicos, econômicose ambientais. Dissertação de Mestrado, Universidade de São Paulo (Programa Interunidades de Pós-Graduação em Energia), São Paulo, SP, Brasil. 